\title{
Effect of disease burden on health-related quality of life in patients with malignant gliomas ${ }^{1}$
}

\author{
David Osoba, ${ }^{2}$ Michael Brada, Michael D. Prados, and W.K. Alfred Yung \\ Department of Medicine, University of British Columbia, Vancouver, British Columbia, Canada (D.O.); \\ Institute of Cancer Research and Royal Marsden NHS Trust, Sutton, Surrey, UK (M.B.); M787 Department of \\ Neurosurgery, University of California, San Francisco, CA 94143 (M.D.P.); Department of Neuro-Oncology, \\ M.D. Anderson Cancer Center, Houston, TX 77030 (W.K.A.Y.)
}

The burden imposed by disease recurrence in patients with high-grade gliomas is not well documented. We studied the frequency of self-report symptoms and the effects on health-related quality of life in patients who had recurrent glioblastoma multiforme or anaplastic astrocytoma and who had a Karnofsky performance score $\geq 70$. Patients completed the European Organization for Research and Treatment of Cancer Quality of Life Questionnaire-Core 30 Items (QLQ-C30) and the Brain Cancer Module (BCM20) before initiation of treatment for first recurrence of disease. Six symptoms (fatigue, uncertainty about the future, motor difficulties, drowsiness, communication difficulties, and headache) were reported with a frequency $>50 \%$ by both groups of patients. An additional two symptoms (visual problems and pain) were also reported with frequencies of $>50 \%$ by patients with recurrent glioblastoma multiforme. Most of the symptoms were likely due to recurrence, but previous radiation therapy and on-going corticosteroid treatment may have also been casual factors for fatigue, whereas uncertainty about the future and pain were probably nonspecific for brain cancer. Problems with motor functioning, vision, leg strength, and pain were reported more frequently by patients with

Received 15 November 1999, accepted 24 May 2000.

${ }^{1}$ Supported in part by Schering-Plough Corp.

${ }^{2}$ Address correspondence and reprint requests to David Osoba, QOL Consulting, 4939 Edendale Ct., West Vancouver, BC, Canada V7W $3 \mathrm{H} 7$.

${ }^{3}$ Abbreviations used are as follows: $\mathrm{AA}$, anaplastic astrocytoma; BCM20, Brain Cancer Module-20 Items; GBM, glioblastoma multiforme; HRQL, health-related quality of life; KPS, Karnofsky Performance score; QLQ-C30, Quality of Life Questionnaire-Core 30 Items. recurrent glioblastoma multiforme than by those with recurrent anaplastic astrocytoma. Scores on health-related quality-of-life functioning scales were similar in the two groups. Finally, the scores for patients who had recurrent high-grade gliomas and a Karnofsky performance score $\geq 70$ were compared with the reported health-related quality of life scores of patients with other cancers. Their scores were similar to those of patients with metastatic cancers and worse than those of patients with localized cancers. Neuro-Oncology 2, 221-228, 2000 (Posted to Neuro-Oncology [serial online], Doc. 99-055, September 15, 2000. URL <neuro-oncology.mc.duke.edu>)

$\mathrm{P}$ rimary brain cancer is a relatively infrequent disease. It was projected that it will be diagnosed in about 16,500 people in the USA compared with an estimated diagnosis of 184,200 new breast cancer cases and an overall 1,220,100 new cancer cases in 1999 (American Cancer Society, 2000; Landis et al., 1999). However, the mortality rate in brain cancer is high and, in 2000, about 13,000 of patients will die from it compared with an estimated mortality of 41,200 for breast cancer. It accounts for one-quarter of all childhood cancers and, after leukemia, is the second leading cause of cancer deaths among children.

Of the primary malignant brain cancers, $60 \%$ are malignant gliomas (Levin et al., 1997). These vary from well-differentiated astrocytomas (grades I and II) comprising $15 \%$ to $20 \%$ of all malignant gliomas, through $\mathrm{AAs}^{3}$ and oligoastrocytomas (grade III) comprising 30\% to $35 \%$, to GBMs (grade IV), which represent $40 \%$ to $50 \%$ of all malignant gliomas. Thus, high-grade gliomas (grades III and IV) comprise the majority $(80 \%-85 \%)$ of all primary brain cancer, with an incidence of about 13,500 to 14,500 per year in the USA. 
Almost all deaths (90\%) from primary brain cancer occur in patients with grade III and IV tumors. Grade IV tumors are the most aggressive, and virtually all patients with grade IV tumors will die from their cancer. Overall, only about $19 \%$ of patients with malignant gliomas will be amenable to complete surgical resection, whereas about $17 \%$ will have a biopsy only, and the remainder $(64 \%)$ will undergo partial resections (Simpson et al., 1993). Consequently, most patients will also require radiation therapy, a modality that improves the 5- and 10-year survival rates in incompletely resected high-grade astrocytomas (Chamberlain and Kormanik, 1998).

Adjuvant chemotherapy may be used also, but improvement in survival is modest (Chamberlain and Kormanik, 1998; Kornblith and Walker, 1988; Levin, 1999; Levin et al., 1990).

Grade III malignant gliomas (AAs) are somewhat less aggressive than grade IV tumors (GBMs). Grade III tumors are difficult to remove completely at surgery and, at recurrence, the tumor often shows higher-grade elements. While median survival is reported to be from 36 to 48 months for grade III tumors, it is about 6 months for grade IV tumors (Levin et al., 1997). Time to progression is longer for grade III tumors (about 9 months) than grade IV tumors (about 4 months). After recurrence, survival times are short, with median survivals being in the range 6 to 10 months for patients with grade III tumors and 2 to 3 months for those with grade IV tumors.

Overall, the diagnosis of high-grade glioma carries a poor prognosis for most patients and, until therapies that markedly improve survival are found, it is important to assess the effects of present therapies on disease burden and health-related quality of life. This will be aided by a prior assessment of the effects of the tumor burden in untreated patients so that a comparative evaluation of the effects of therapy can be made.

\section{Disease Burden}

The term "disease burden" is used to encompass the symptoms and limitations in physical functioning and emotional well-being imposed by the illness. At initial presentation, the most common symptoms may occur singly, or as a constellation, and include headache, anorexia, nausea, vomiting (particularly in children), seizures, sleeping longer at night and drowsiness with napping during the day (Levin et al., 1997). Most of these symptoms are secondary to increased intracranial pressure and may occur at initial presentation or at recurrence after treatment intended to cure or to produce tumor reduction. Aside from surgery, the most commonly used strategy to decrease intracranial pressure is the administration of corticosteroids for intracranial edema. A proportion of patients may remain on corticosteroid therapy for a prolonged time, and this may mask some of the early symptoms and signs of recurrence. Corticosteroids may reduce the severity of the symptoms or reduce their impact, but may impose symptoms of their own, e.g. fluid retention, weight gain, muscle weakness, and Cushingoid appearance.
Neurological deficits that are found at presentation and which may persist after therapy, or occur at recurrence, include motor and sensory deficits, i.e. hemiplegia or speech and visual difficulties. In addition, there may be personality changes, mood disturbances, or decrease in mental capacity and concentration (Meyers et al., 1998, 2000; Weitzner, 1999). The neuropsychiatric and neurocognitive defects may prove to be as, or more, burdensome to patients and their immediate caregivers than some of the neurologic deficits referred to above.

The disease burden, depending on its varying impact on the patients, will have varying effects on overall wellbeing and HRQL. The remainder of this paper will deal with how HRQL is measured and what the effects of the disease are on HRQL.

\section{Health-Related Quality of Life in Brain Cancer}

The ultimate goal of health care is to restore or preserve HRQL (Osoba, 1999). The clinical management of brain cancer is particularly concerned with preserving or restoring neurologic functioning and the amelioration of symptoms arising from decrements in neurologic functioning. Thus, measurement of HRQL to determine whether these goals are being achieved is of paramount importance, and the restoration/improvement of HRQL should be a primary outcome (end point) in high-grade neurologic malignancies.

There are many definitions of HRQL, but simply put, it is the impact of a disease and its treatment on a person's self-assessed ability to function in at least the physical, cognitive, emotional, and social domains of day-to-day life. There are many determinants of physical, cognitive, emotional, and social functioning (e.g. neurologic or anatomic deficits, personality traits, symptom burden, etc.). The intent of HRQL assessment is not to measure the determinants, but rather to scientifically measure the functional outcome by which these determinants are manifested in domains such as physical, emotional, cognitive, and social functioning and in symptom complexes such as fatigue, pain, and other bodily discomfort.

HRQL is measured, most commonly, by reliable and valid self-report questionnaires (instruments). In oncology, it is suggested that a general, cancer-oriented questionnaire be supplemented by a disease and/or a situation-specific questionnaire (module) developed for the clinical trial or clinical situation (Aaronson et al., 1988; Cella et al., 1995). This modular approach has been used for measuring HRQL for brain cancer by at least two sets of questionnaires, the European Organization for Research and Treatment of Cancer core questionnaire (QLQ-C30) supplemented by the BCM20 (Osoba et al., 1996, 1997, 1998a, 2000a, 2000b; Yung et al., 1999a) and by the Functional Assessment of Cancer Therapy-Brain (FACT-Br) questionnaire (Weitzner et al., 1995). A possible major difference between these two sets of questionnaires is that the 20 items of the BCM20 are grouped into 4 subscales dealing with symptom domains and an additional 7 single items, whereas the Functional Assessment of Cancer Therapy-Brain groups all of its 19 items into a single subscale. These two approaches to measuring HRQL in brain cancer have not been tested 
simultaneously in a single group of patients to determine whether meaningful differences between them exist and, if so, whether the differences are clinically important.

\section{The Burden of Brain Cancer on HRQL}

There are several aspects to consider when determining the burden (symptoms and neurologic deficits) of brain cancer on HRQL. These include the histologic type of cancer, the time in the illness trajectory, (i.e., just after the diagnosis and before or after surgery/radiation therapy), the response of the cancer to these primary therapies, the time of recurrence, and the effects of chemotherapy.

One aspect of the disease burden that needs to be more fully documented is the type and frequency of selfreported symptoms, particularly at the time of recurrence. In the studies reported here, we determined the frequency of symptoms reported by patients who completed the QLQ-C30 and BCM20 at the time of first recurrence and after previous treatment, consisting of surgery and radiation therapy, had been given. We also determined the effect of initial histology on HRQL and, finally, compared the scores from these patients with high-grade gliomas to those of patients with other localized or metastatic cancers.

\section{Materials and Methods}

\section{Patients}

The data were derived from 3 separate clinical trials involving a total of 525 registered patients with either GBM $(n=363)$ or AA $(n=162)$ at first recurrence after surgery and radiation therapy. These patients were about to undergo chemotherapy with either temozolomide or procarbazine. All patients were at least 18 years old and had a KPS $\geq 70$ at the time of entry into the study. There was unequivocal evidence of tumor recurrence/progression by Gd-MRI or contrast-enhanced CT scanning. All had histologic confirmation of tumor histology at the time of recurrence. The HRQL results from these clinical trials have been reported elsewhere (Brada et al., 1999; Osoba et al., 1997, 1998a, 1999a, 2000a, 2000b; Prados et al., 1999; Yung et al., 1999a; Yung et al., 1999b).

\section{HRQL Assessments}

HRQL status was assessed by the European Organization for Research and Treatment of Cancer QLQ-C30 (+3) (Fayers et al., 1999) a later version of the European Organization for Research and Treatment of Cancer QLQ-C30 (Aaronson et al., 1993) that was used to develop version 2.0 (Osoba et al., 1997), and the BCM20 (Osoba et al., 1996). Both are reliable and valid instruments in the setting of recurrent high-grade gliomas (Osoba et al., 1996, 1997). The QLQ-C30 (+3) consists of 33 items that form 5 functioning domains (physical, role, emotional, social, and cognitive), a global QL/overall health domain, 3 symptom domains (fatigue, pain, nausea and vomiting), and 6 single items (dyspnea, diarrhea, constipation, anorexia, insomnia, and financial impact). The version 2.0 scoring system (Fayers et al., 1999) was used in these studies, and the instrument will be referred to as version 2.0 in the remainder of this report. The BCM 20 contains 20 items grouped into 4 domains (future uncertainty, visual disorder, communication deficit, and motor dysfunction) and 7 single items (headache, seizure, drowsiness, hair loss, itching, weakness of both legs, and difficulty controlling bladder function). Some of these symptoms, (i.e. hair loss and itching) were related to toxicity of previous treatment by radiation therapy or side effects of temozolomide, whereas the remainder are associated with the recurrence of GBM (Osoba et al., 1999b).

The HRQL questionnaires (in the appropriate languages) were given to patients for completion within 7 days (baseline assessment) of the planned onset of chemotherapy for first recurrence after initial treatment by surgery and radiation therapy.

\section{Statistical Procedures}

The QLQ-C30 was scored according to methods described in the 2nd edition of the European Organization for Research and Treatment of Cancer's QLQ-C30 Scoring Manual (Fayers et al., 1999). All raw scores were converted to lie in a range between 0-100. For the functioning scales and the global QL scale, a higher score indicates better functioning, whereas for the symptom scales/items a higher score indicates more of the symptom or difficulty. The BCM20 was scored in a manner analogous to the QLQ-C30 (version 2.0) (Osoba et al., 1996): Higher scores indicate more of the symptom or more difficulty.

A clinically significant difference in scores was defined as a change of $\geq 10$ (on a scale of 0-100) lasting for at least two HRQL assessments 4 weeks apart. This criterion was based on previous studies involving a formal assessment of how much change in QLQ-C30 scores is perceptible to patients (subjective significance) (Osoba et al., 1998b), and how much change is associated with changes in disease status (King, 1996). Other approaches, using other questionnaires in other illnesses, also indicate that a difference of $\geq 10$ (on a $0-100$ scale) may be interpreted as being clinically significant (Jaeschke et al., 1989; Juniper et al., 1994; Ware et al., 1994). Therefore, on symptom scales and single items, we used scores of $\geq 10$ as being significant to patients, because patients experienced the symptom as perceptible and different from having none of the symptoms, i.e., a score of 0 .

\section{Results}

\section{Patient Characteristics}

Following are the main characteristics of the 488 patients (93\% of registered patients completing baseline HRQL data). The ages for GBM patients ranged from 23 to 77 years with a mean of 51.2 years, whereas ages for the AA patients ranged from 19 to 76 years with a mean of 42.5 years. In the GBM group, $63.2 \%$ of the patients were male, and in the AA group, $56.5 \%$ were male. Most of 
the patients $(96 \%)$ were white. The percentages of patients with the following KPS scores in the GBM and AA groups, respectively, were 100 (5.9\%, 16.7\%), 90 $(32.3 \%, 31.2 \%), 80(27.8 \%, 23.2 \%)$, and $70(33.7 \%$, $28.3 \%)$. In summary, the AA patients were a little younger than the GBM patients, and the proportion of AA patients with a KPS of 100 was smaller than that of the GBM group showing a KPS of 100 .

\section{Symptom Burden}

Eight of the symptoms occurred with a frequency of $>50 \%$ in patients with GBM, while patients with AA reported 6 symptoms with this frequency (Table 1). The 6 most frequently reported symptoms were fatigue, uncertainty about the future, motor dysfunction, drowsiness, communication deficit, and headache in both groups. In addition, several other symptoms were reported by $<50 \%$ of patients. The frequency of symptoms was generally similar in both groups of patients, but motor dysfunction, visual disorder, leg weakness, and pain were less frequent in patients with AA.

\section{Newly Diagnosed Versus Recurrent Disease in GBM}

During the development of the BCM20, 105 patients who had GBM, in differing phases of the illness, were assessed for HRQL (Osoba et al., 1996). Forty-one patients were recently diagnosed and 64 had recurrent disease. Compared with patients who had newly diagnosed disease, those with recurrent disease had significantly more difficulties involving motor dysfunction (13.0 versus $26.7, P<0.01$ ), communication deficit $(15.2$ versus $28.7, P<0.01$ ), weakness of both legs (4.1 versus 12.5, $P<0.05)$, and trouble controlling bladder function (4.1 versus 17.2. $P<0.01$ ) (Osoba et al., 1999a).

In the more recent studies, when patients with recurrent GBM were enrolled in temozolomide therapy trials (Yung et al., 1999b), baseline (pretreatment) HRQL scores were similar to those with recurrent disease in the BCM20 development study. Thus, the neurologic deficits are greater in patients with recurrent disease than in patients with newly diagnosed GBM.

\section{Proportions of Patients With GBM and AA Reporting Less Than Optimum Baseline Scores}

The mean baseline HRQL scores, including all the functioning scales and symptom scales for patients with recurrent GBM and AA in the above studies, are shown in Table 2. The functioning scale scores are similar between the various groups. However, when the proportions of patients with scores of 90 or less in the functioning domains are compared, the tendency for more patients with GBM than for patients with AA to report scores of 80 or less for physical, role, and possibly cognitive functioning is evident (Table 3). Nevertheless, the overall conclusion is that HRQL functioning scores at recurrence are not strikingly different regardless of the histology of the original tumor. It is possible that some of this similarity is accounted for by the selection of patients with a KPS $\geq 70$ for these studies.
Table 1. Frequencies of the most commonly reported symptoms with a magnitude $\geq 10$ (0-100 scale) in patients with recurrent glioblastoma multiforme and recurrent anaplastic astrocytoma with a Karnofsky performance score of $\geq 70$

\begin{tabular}{lcc} 
& \% in GBM & $\%$ in AA \\
\hline Most frequent symptoms & 94 & 89 \\
Fatigue $^{\mathrm{a}}$ & 81 & 85 \\
Future uncertainty $^{\mathrm{a}}$ & 89 & 73 \\
Motor dysfunction & 79 & 69 \\
Drowsiness $_{\text {Communication deficit }}$ & 69 & 66 \\
Visual disorder $_{\text {Pain }}^{\mathrm{a}}$ & 59 & 44 \\
Headache $_{\text {Other symptoms }}$ & 55 & 25 \\
Weak legs $^{\mathrm{a}}$ & 52 & 52 \\
Bladder control problems $_{\text {Hair loss }}^{\mathrm{a}}$ & & \\
Nausea/vomiting $_{\text {Itching }}^{\mathrm{a}}$ & 43 & 29 \\
Seizures & 33 & 28 \\
\hline
\end{tabular}

Abbreviations: GBM, glioblastoma multiforme; $A A$, anaplastic astrocytoma.

${ }^{a}$ May not be due entirely to recurrent brain cancer.

\section{HRQL Scores and KPS Scores}

As previously shown by others (Mackworth et al., 1992), we also found that KPS scores were poorly correlated with baseline HRQL scores in patients with recurrent AA and GBM (data not shown).

\section{Comparison of Baseline Scores in High-Grade Gliomas With Those of Other Cancers}

Baseline QLQ-C30 scores of patients with recurrent high-grade gliomas were compared with baseline scores of patients with other cancers (Table 4) (Aaronson et al., 1993; Fayers et al., 1998; King, 1996; Osoba et al., 1994, 1999b). Generally, the HRQL scores of patients with recurrent high-grade glioma were similar to those of patients with advanced ovarian and lung cancer and those of patients with metastatic heterogeneous cancers, except that the patients with recurrent brain cancer had worse cognitive functioning and less pain. Patients with metastatic, hormone-resistant prostate cancer and those with lung cancer sustaining more than $10 \%$ weight loss reported worse HRQL scores than did patients with recurrent brain cancer. However, groups of patients with localized cancer or with no evident disease had better HRQL scores than those with recurrent high-grade gliomas. Normative data from the population chosen at random in Denmark (Klee et al., 1997) and Norway (Hjermstad et al., 1998) also confirm that scores in patients with recurrent brain cancer were much lower than those in the general population. 
Table 2. Mean baseline health-related quality-of-life scores in patients with glioblastoma multiforme and anaplastic astrocytoma

\begin{tabular}{|c|c|c|c|c|}
\hline \multirow[b]{2}{*}{ QLQ-C30 } & \multirow{2}{*}{$\frac{\text { GBM }(n=105-109)}{\text { Temozolomide }^{\mathrm{a}}}$} & \multicolumn{2}{|c|}{$\mathrm{GBM}(n=172-179)$} & \multirow{2}{*}{$\begin{array}{c}\text { AA }(n=139-145 \\
\text { Temozolomide }^{a} \\
\text { Mean }(+S D)\end{array}$} \\
\hline & & $\begin{array}{c}\text { Temozolomide }^{\mathrm{a}}(n=86-89) \\
\text { Mean }(+\mathrm{SD})\end{array}$ & $\begin{array}{c}\text { Procarbazine }^{\mathrm{a}}(n=86-90) \\
\text { Mean }(+\mathrm{SD})\end{array}$ & \\
\hline \multicolumn{5}{|l|}{ Functioning scales } \\
\hline Physical & $67(29)$ & $72(29)$ & $68(29)$ & $73(31)$ \\
\hline Role & $55(33)$ & $58(32)$ & $61(28)$ & $67(32)$ \\
\hline Emotional & $63(26)$ & $70(19)$ & $69(21)$ & $71(20)$ \\
\hline Cognitive & $62(31)$ & $62(29)$ & $64(26)$ & $69(28)$ \\
\hline Social & $64(34)$ & $60(32)$ & $61(29)$ & $64(31)$ \\
\hline Global GL & $56(23)$ & $63(21)$ & $59(23)$ & $61(23)$ \\
\hline \multicolumn{5}{|l|}{ Symptom scales } \\
\hline Fatigue & $39(22)$ & $40(23)$ & $43(24)$ & $37(24)$ \\
\hline Nausea/vomiting & $6(15)$ & $5(12)$ & $7(14)$ & $9(20)$ \\
\hline Pain & $15(23)$ & $16(21)$ & $22(22)$ & $17(24)$ \\
\hline Dyspnea & $13(20)$ & $15(20)$ & $17(22)$ & $8(20)$ \\
\hline Insomnia & $22(31)$ & $23(29)$ & $23(27)$ & $21(26)$ \\
\hline Anorexia & $9(20)$ & $7(25)$ & $15(25)$ & $15(26)$ \\
\hline Constipation & $14(24)$ & $9(26)$ & $18(28)$ & $14(23)$ \\
\hline Diarrhea & $7(15)$ & $8(18)$ & $7(16)$ & $8(19)$ \\
\hline Financial impact & $23(32)$ & $38(35)$ & $32(33)$ & $30(35)$ \\
\hline \multicolumn{5}{|l|}{$\mathrm{BCM} 20$} \\
\hline Future uncertainty & $40(29)$ & $44(28.2)$ & $41(26)$ & $41(26)$ \\
\hline Visual disorder & $20(24)$ & $22(27.7)$ & $18(23)$ & $14(21)$ \\
\hline Motor dysfunction & $26(26)$ & $33(26.0)$ & $28(24)$ & $26(26)$ \\
\hline Communication deficit & $21(31)$ & $35(33.4)$ & $26(25)$ & $24(26)$ \\
\hline Headaches & $19(26)$ & $26(30.9)$ & $27(27)$ & $26(32)$ \\
\hline Seizures & $14(28)$ & $12(25.4)$ & $56(17)$ & $11(24)$ \\
\hline Drowsiness & $33(28)$ & $41(27.7)$ & $40(26)$ & $34(30)$ \\
\hline Hair loss & $18(30)$ & $16(27.2)$ & $14(26)$ & $21(34)$ \\
\hline Itching & $9(21)$ & $14(24.6)$ & $14(23)$ & $14(27)$ \\
\hline Weakness of legs & $23(31)$ & $19(25.7)$ & $20(29)$ & $16(29)$ \\
\hline Bladder control & $17(27)$ & $17(27.3)$ & $12(21)$ & $16(29)$ \\
\hline
\end{tabular}

Abbreviations: See Table 1; QL, quality of life.

${ }^{\mathrm{a}}$ Treatment assignment.

\section{Discussion}

High-grade gliomas are difficult to cure and often recur after primary therapy with surgery and radiation therapy. Thus, until better primary therapy is devised, it is necessary to deal with recurrent disease as well as possible. Under these circumstances, the HRQL of patients with high-grade glioma is a very important consideration in the overall management of the patient.

Very little evidence-based data is available about the HRQL of patients with newly diagnosed GBM or AA. However, much more information has become available about HRQL in patients with recurrent high-grade glioma, mainly as a result of the analysis of a large database from patients about to undergo chemotherapy with temozolomide or procarbazine. Some of the results of the HRQL assessment in these clinical trials have been reported (Brada et al., 1999; Osoba et al., 1997, 1998a, 1999a, 2000a, 2000b; Prados et al., 1999; Yung et al., 1999b). A
Table 3. Frequency of baseline scores $\leq 90$ in QLQ-C30 functioning scales in recurrent glioblastoma multiforme versus anaplastic astrocytoma

\begin{tabular}{lccc} 
Functioning scale & Score & \%GBM & \%AA \\
\hline Physical & $\leq 80$ & 51 & 39 \\
Role & $\leq 90$ & 68 & 57 \\
& $\leq 80$ & 68 & 54 \\
Emotional & $\leq 90$ & 84 & 68 \\
& $\leq 80$ & 67 & 65 \\
Cognitive & $\leq 90$ & 80 & 79 \\
& $\leq 80$ & 60 & 51 \\
Social & $\leq 90$ & 85 & 76 \\
& $\leq 80$ & 63 & 58 \\
Overall health/global QL & $\leq 90$ & 79 & 73 \\
& $\leq 80$ & 80 & 76 \\
& $\leq 90$ & 93 & 89 \\
\hline
\end{tabular}

Abbreviations: See Tables 1 and 2. 
Table 4. Mean health-related quality-of-life scores in recurrent high-grade gliomas compared with other cancers

\begin{tabular}{|c|c|c|c|c|c|c|c|c|c|}
\hline QLQ-C30 & $\begin{array}{l}\text { Recurrent } \\
\text { high-grade } \\
\text { gliomas }\end{array}$ & $\begin{array}{c}\text { Advanced } \\
\text { ovary }\end{array}$ & $\begin{array}{l}\text { Advanced } \\
\text { lung }\end{array}$ & $\begin{array}{l}\text { Matastic } \\
\text { melanoma }\end{array}$ & $\begin{array}{l}\text { Heterogenous } \\
\text { metastic }\end{array}$ & $\begin{array}{c}\text { Advanced } \\
\text { prostate }\end{array}$ & $\begin{array}{l}>10 \% \\
\text { Weight } \\
\text { loss, lung }\end{array}$ & $\begin{array}{l}\text { Localized } \\
\text { heterogenous }\end{array}$ & $\begin{array}{c}\text { Majority } \\
(86 \%) \\
\text { with NED }\end{array}$ \\
\hline \multicolumn{10}{|l|}{ Functioning scales } \\
\hline Physical & 70 & 66 & 69 & 67 & 69 & 52 & 47 & 80 & 86 \\
\hline Role & 60 & 52 & 66 & 60 & 60 & 45 & 40 & 74 & 84 \\
\hline Emotional & 68 & 69 & 67 & 77 & 68 & 69 & 67 & 70 & 80 \\
\hline Cognitive & 62 & 62 & 66 & 67 & 63 & 59 & 67 & 75 & 82 \\
\hline Social & 64 & 81 & 84 & - & 83 & 76 & - & 84 & 85 \\
\hline Global GL & 60 & 54 & 55 & 60 & 54 & 45 & 43 & 64 & 76 \\
\hline \multicolumn{10}{|l|}{ Symptom scales } \\
\hline Fatigue & 40 & 43 & 39 & 43 & 39 & 50 & 63 & 32 & 25 \\
\hline Pain & 18 & 33 & 31 & 30 & 33 & 51 & - & 23 & 17 \\
\hline Nausea/vomiting & 7 & 5 & 4 & 17 & 5 & 18 & 13 & 4 & 5 \\
\hline
\end{tabular}

Abbreviations: NED, no evident disease; - , no data; QL, quality of life.

full report of the clinical results of the trial for patients with AA treated with temozolomide has also been reported (Yung et al., 1999a). However, little information has been published on the potential differences in HRQL at recurrence relative to newly diagnosed disease, the frequency and severity of symptoms (symptom burden), or the effects of tumor histology and baseline HRQL of patients with high-grade gliomas compared to patients with other types of cancer. This report addresses these questions by examining the baseline HRQL data from $93 \%$ of the patients registered on the previously mentioned trials.

Of the 14 most frequently reported symptoms by patients filling in the HRQL questionnaire at the time of recurrence, fatigue, future uncertainty, motor dysfunction, drowsiness, communication deficit, visual disorder pain, and headache were all reported with a frequency of $>50 \%$ in patients with recurrent GBM. Some symptoms may have been secondary to treatment (hair loss, itching from radiation therapy, and weakness of the legs from corticosteroids), and some were probably nonspecific for brain cancer (uncertainty about the future and pain), but others are probably due to the primary disease. In AA patients, all symptoms except visual disorder and pain were also reported with a frequency of $>50 \%$ at the time of recurrence. Thus, it appears that these patients carry a significant symptom burden, although it is difficult to find a similar, well-described cohort of patients in the literature for newly diagnosed high-grade gliomas. Available literature on the HRQL scores of patients with other cancers indicates that patients with recurrent high-grade gliomas are similar to patients with advanced and metastatic cancers rather than patients with localized cancers. These data, taken together, make it evident that patients with recurrent high-grade gliomas not only carry a high symptom burden, but have very significant deficits in their ability to function in several other domains of life, as well.

As might be expected, neurologic deficits, as reported by GBM patients, are greater at the time of recurrence than at the time of initial diagnosis. It seems reasonable to postulate that many of the neurologic deficits at the time of diagnosis were only partially resolved in these patients and that recurrence adds to the previous problems. However, a longitudinal study of HRQL from the time of presentation through treatment and recurrence will need to be carried out to provide a definitive answer.

One of the concerns of investigators in this field is the interpretation of the reliability of the answers provided by patients with less than average cognitive functioning. The data in this report indicate that these patients' self-reports of cognitive functioning indicate lower scores than those reported by other patients with other cancers, whether localized or metastatic. These lower scores were for patients with KPS scores of 70 or higher, and cognitive functioning scores would be expected to be even lower in patients with lower KPS scores. The reliability of the responses becomes particularly important in longitudinal studies where cognitive function would be expected to deteriorate even further with time. To address this issue, some studies have examined the use of questionnaires that are intended to be completed by proxies, i.e., observers (Sneeuw et al., 1997a). One logical way of using proxy ratings in brain cancer is to obtain both proxy and self ratings as early as possible in the trajectory of the disease and, then, as the patient's ability to respond deteriorates, to rely mainly on the proxy ratings. If there were good concordance between the proxy and patient ratings early in the illness, then the proxy ratings would likely be reliable at a later time when the patients' ratings were no longer reliable. The use of simultaneous proxy and patient ratings also provides a training period during which the proxy becomes attuned to the patients concerns and condition. It has been found that there is a discrepancy between proxy and patient ratings in highly subjective domains such as emotional functioning, pain, and fatigue. Proxies tend to overestimate the patients' emotional symptoms but to underestimate pain and fatigue. Nevertheless, workers in this area have concluded that the benefits of proxy ratings of HRQL outweigh their limitations (Sneeuw et al., 1997a, 1997b).

In summary, HRQL can be measured and gives meaningful results in high-grade gliomas. Recurrence of 
disease has a significant deleterious impact on patients' lives. The effect of treatment aimed at reducing and abrogating the deleterious effects should be measured and used in addition to survival as an outcome parameter. Therapy that is successful in improving or maintaining HRQL may be concluded to be worthwhile despite the absence of significant improvement in survival.

\section{Acknowledgments}

We are grateful to Wayne Weng of Schering-Plough for providing the data for these studies and for helpful advice throughout the projects. We also thank Jill Vardy for assistance with assembling the manuscript and ScheringPlough Corporation for financial support.

\section{References}

Aaronson, N.K., Bullinger, M., and Ahmedzai, S. (1988) A modular approach to quality-of-life assessment in cancer clinical trials. Recent Results Cancer Res. 111, 231-249.

Aaronson, N.K., Ahmedzai, S., Bergman, B., Bullinger, M., Cull, A., Duez, N.J., Filiberti, A., Flechtner, H., Fleischman, S.B., de Haes, J.C.J.M., Kaasa, S., Klee, M., Osoba, D., Razavi, D., Rofe, P.B., Schraub, S., Sneeuw, K., Sullivan, M., and Takeda, F., for the European Organization for Research and Treatment of Cancer Study Group on Quality of Life (1993) The European Organization for Research and Treatment of Cancer QLQ-C30: A qualityof-life instrument for use in international clinical trials in oncology. J. Natl. Cancer Inst. 85, 365-376.

American Cancer Society (2000), September 5, 2000. Cancer facts and figures 2000: Graphical data. URL http://www.cancer.org/statistics/cff99data/html.

Brada, M., Yung, W.K.A., and Osoba, D. (1999) Symptom burden in patients (PTS) with recurrent high-grade gliomas. Proc. Am. Soc. Clin. Oncol. 18, 147a. (Abstract)

Cella, D.F., Bonomi, A.E., Lloyd, S.R., Tulsky, D.S., Kaplan, E., and Bonomi, P. (1995) Reliability and validity of the Functional Assessment of Cancer Therapy-Lung (FACT-L) quality of life instrument. Lung Cancer 12, 199220.

Chamberlain, M.C., and Kormanik, P.A. (1998) Practical guidelines for the treatment of malignant gliomas. West. J. Med. 168, 114-120.

Fayers, P.M., Aaronson, N.K., Bjordal, K., and Sullivan, M., on behalf of the EORTC Quality of Life Study Group (1995) EORTC QLQ-C30 Scoring Manual. Brussels, Belgium: EORTC Data Centre.

Fayers, P.M., Weeden, S., and Curran, D., on behalf of the EORTC Quality of Life Study Group (1998) EORTC QLQ-C30 Reference Values. Brussels, Belgium: EORTC Data Centre.

Fayers, P.M., Aaronson, N.K., Bjordal, K., Curran, D., and Groenvold, M., on behalf of the EORTC Quality of Life Study Group (1999) EORTC QLQC30 Scoring Manual. Second ed. Brussels, Belgium: EORTC Data Centre.

Hjermstad, M.J., Fayers, P.M., Bjordal, K., and Kaasa, S. (1998) Health-related quality of life in the general Norwegian population assessed by the European Organization for Research and Treatment of Cancer Core Qualityof-Life Questionnaire: The QLQ-C30 (+3). J. Clin. Oncol. 16, 1188-1196.

Jaeschke, R., Singer, J., and Guyatt, G.H. (1989) Measurement of health status: Ascertaining the minimal clinically important difference. Control. Clin. Trials 10, 407-415.

Juniper, E.F., Guyatt, G.H., Willan, A., and Griffith, L.E. (1994) Determining a minimal important change in a disease-specific Quality of Life Questionnaire. J. Clin. Epidemiol. 47, 81-87.

King, M.T. (1996) The interpretation of scores from the EORTC quality of life questionnaire QLQ-C30. Qual. Life Res. 5, 555-567.

Klee, M., Groenvold, M., and Machin, D. (1997) Quality of life of Danish women: Population-based norms of the EORTC QLQ-C30. Qual. Life Res. 6, 27-34.

Kornblith, P.L., and Walker, M. (1988) Chemotherapy for malignant gliomas. J. Neurosurg. 68, 1-17.

Landis, S.H., Murray, T., Bolden, S., and Wingo, P.A. (1999) Cancer statistics, 1999. CA. Cancer J. Clin. 49, 8-31.
Levin, V.A. (1999) Neuro-oncology: An overview. Arch. Neurol. 56, 401-404. Levin, V.A., Silver, P., Hannigan, J., Wara, W.M., Gutin, P.H., Davis, R.L., and Wilson, C.B. (1990) Superiority of post-radiotherapy adjuvant chemotherapy with $C C N U$, procarbazine, and vincristine (PCV) over BCNU for anaplastic gliomas: NCOG $6 \mathrm{G} 61$ final report. Int. J. Radiat. Oncol. Biol. Phys. 18, 321-324.

Levin, V.A., Leibel, S.A., and Gutin, P.H. (1997) Neoplasms of the central nervous system. In: DeVita, V.T., Jr, Hellman, S., and Rosenberg, S.A. (Eds.), Cancer : Principles and Practices of Oncology. Fifth ed. Philadelphia, PA: Lippincott-Raven. pp. 2022-2082.

Mackworth, N., Fobair, P., and Prados, M.D. (1992) Quality of life self-reports from 200 brain tumor patients: Comparisons with Karnofsky performance scores. J. Neurooncol. 14, 243-253.

Meyers, C.A., Weitzner, M.A., Valentine, A.D., and Levin, V.A. (1998) Methylphenidate therapy improves cognition, mood, and function of brain tumor patients. J. Clin. Oncol. 16, 2522-2527.

Meyers, C.A., Hess, K.R., Yung, W.K., and Levin, V.A. (2000) Cognitive function as a predictor of survival in patients with recurrent malignant glioma. J. Clin. Oncol. 18, 646-650.

Osoba, D. (1999) What has been learned from measuring health-related quality of life in clinical oncology. Eur. J. Cancer 35, 1565-1570.

Osoba, D., Zee, B., Pater, J., Warr, D., Kaizer, L., and Latreille, J. (1994) Psychometric properties and responsiveness of the EORTC Quality of Life Questionnaire (QLQ-C30) in patients with breast, ovarian and lung cancer. Qual. Life Res. 3, 353-364.

Osoba, D., Aaronson, N.K., Muller, M., Sneeuw, K., Hsu, M-A.,Yung, W.K.A., Brada, M., and Newlands, E. (1996) The development and psychometric validation of a brain cancer quality-of-life questionnaire for use in combination with general cancer-specific questionnaires. Qual. Life Res. 5, 139150.

Osoba, D., Aaronson, N.K., Muller, M., Sneeuw, K., Hsu, M-A.,Yung, W.K.A., Brada, M., and Newlands, E. (1997) Effect of neurological dysfunction on health-related quality of life in patients with high-grade glioma. J. NeuroOncol. 34, 263-278.

Osoba, D., Levin, V., Yung, W.K.A., Brada, M., Prados, M., Albrecht, R., Olson, J., Weng, C.S.W., and Dugan, M. (1998a) Health-related quality of life (HRQL) benefits in patients with recurrent anaplastic astrocytoma (AA) treated with temozolomide (TEM). Proc. Am. Soc. Clin. Oncol. 17, 388a. (Abstract)

Osoba, D., Rodrigues, G., Myles, J., Zee, B., and Pater, J. (1998b) Interpreting the significance of changes in health-related quality-of-life-scores. J. Clin. Oncol. 16, 139-144.

Osoba, D., Brada, M., and Yung, W.K.A. (1999a) Health-related quality of life (HRQL) benefits of treatment with temozolomide (TMZ) vs procarbazine (PCB) in patients (PTS) with glioblastoma multiforme (GBM). Proc. Am. Soc. Clin. Oncol. 18, 141a. (Abstract)

Osoba, D., Tannock, I.F., Ernst, D.S., and Neville, A.J. (1999b) Health-related quality of life in men with metastatic prostate cancer treated with prednisone alone or mitoxantrone and prednisone. J. Clin. Oncol. 17, 16541663. 
Osoba, D., Brada, M., Yung, W.K.A., and Prados, M. (2000a) Health-related quality of life in patients treated with temozolomide versus procarbazine for recurrent glioblastoma multiforme. J. Clin. Oncol. 18, 1481-1491.

Osoba, D., Brada, M., Yung, W.K.A., and Prados, M. (2000b) Health-related quality of life in patients with anaplastic astrocytoma during treatment with temozolomide. Eur. J. Cancer, in press.

Prados, M., Yung, A., Chang, S., Delattre, J-Y, Yaya-Tur, R., Rosenfeld, S., Brada, M., Friedman, H., Albright, R., Olsen, J., Bruner, J., Yu, N., Dugan, M., and Levin, V. (1999) A phase-2 trial of Temodal (temozolomide) in patients with anaplastic astrocytoma at first relapse. Proc. Am. Soc. Clin. Oncol. 18, 139a. (Abstract)

Simpson, J.R., Horton, J., Scott, C., Curran, W.J., Rubin, P., Fischbach, J., Isaacson, S., Rotman, M., Asbell, S.O., Nelson, J.S., Weinstein, A.S., and Nelson, D.F. (1993) Influence of location and extent of surgical resection on survival of patients with glioblastoma multiforme: Results of three consecutive Radiation Therapy Oncology Group (RTOG) clinical trials. Int. J. Radiat. Oncol. Biol. Phys. 26, 239-244.

Sneeuw, K.C.A., Aaronson, N.K., Osoba, D., Muller, M..J., Hsu, M.-A., Yung, W.K.A., Brada, M., and Newlands, E.S. (1997a) The use of significant others as proxy raters of the quality of life of patients with brain cancer. Med. Care 35, 490-506.

Sneeuw, K.C.A., Aaronson, N.K., Sprangers, M.A.G., Detmar, S.B., Wever,
L.D., and Schornagel, J.H. (1997b) Value of caregiver ratings in evaluating the quality of life of patients with cancer. J. Clin. Oncol. 15, 1206-1217.

Ware, J.E., Jr., Kosinski, M., and Keller, S.D. (1994) SF-36 Physical and Mental Health Summary Scales: A User's Manual. Boston, Mass.: The Health Institute, New England Medical Center.

Weitzner, M.A. (1999) Psychosocial and neuropsychiatric aspects of patients with primary brain tumors. Cancer Invest. 17, 285-291.

Weitzner, M.A., Meyers, C.A., Gelke, C.K., Byrne, K.S., Cella, D.F., and Levin, V.A. (1995) The Functional Assessment of Cancer Therapy (FACT) Scale: Development of a brain subscale and revalidation of the general version (FACT-G) in patients with primary brain tumors. Cancer 75, 1151-1161.

Yung, W.K.A., Prados, M.D., Yaya-Tur, R., Rosenfeld, S.S., Brada, M., Friedman, H.S., Albright, R., Olson, J., Chang, S.M., O'Neill, A.M., Friedman, A.H., Bruner, J., Yue, N., Dugan, M., Zaknoen, S., and Levin, V.A. (1999a) Multicenter phase II trial of temozolomide in patients with anaplastic astrocytoma or anaplastic oligoastrocytoma at first relapse. J. Clin. Oncol. $17,2762-2771$.

Yung, A., Levin, V.A., Albright, R., Olson, J., Fredericks, R., Fink, K., Prados, M., Brada, M., Spence, A., Brunner, J., Yue, N., Dugan, M.H., and Zaknoen, S. (1999b) Randomized trial of Temodal (TEM) vs procarbazine (PCB) in glioblastoma multiforme (GBM) at first relapse. Proc. Am. Soc. Clin. Oncol. 18, 139a. (Abstract) 